Proc. Indian Acad. Sci. (Chem. Sci.), Vol. 89, Number 2, April 1980, pp. 109-118. (C) Printed in India.

\title{
A theoretical study of the Jorgensen's nephelauxetic effect in some simple transition metal halo-complexes from a molecular orbital computation
}

\author{
S VIJAYA ${ }^{+}$and P T MANOHARAN \\ Department of Chemistry, Indian Institute of Technology, Madras 600 036, India \\ + Present address : University of Southern California, Department of Chemistry, \\ University Park, Los Angeles, California 90007, USA
}

MS received 29 August 1979 ; revised 16 January 1980

\begin{abstract}
A theoretical study of Jorgensen's nephelauxetic effect in some simple high spin transition metal halo-complexes has been made with an unrestricted HartreeFock-SCF-INDO MO calculation. The nephelauxetic spectroscopic parametersthe Racah integral $B$ and the covalency factor $\beta$ - have been theoretically evaluated from the above MO analysis results. For this a pseudo-atom model and an eigenMO model have been employed. The results allow a comparison to be made between the experimental and theoretical results, which indicate the relative success of the pseudo-atom model over the other. It has also been pointed out that the results from both models would have improved considerably if the use of metal valence basis orbitals corresponding to its final oxidation state had been resorted to. The concept of differential covalency and its effect on nephelauxetic parameters are also discussed.
\end{abstract}

Keywords. Nephelauxetic effect ; metal-halo-complexes ; Jorgensen effect ; molecular orbital computation; pseudo-atom model.

\section{Introduction}

The theory of atomic spectra provides approximate expressions (Slater 1960; Condon and Shortley 1959 ; Griffith 1971) for the energy terms of the transition atoms and ions in their various configurations as functions of the coulomb and exchange energies represented by Slater-Condon integrals $F_{0}, F_{k}$ and $G_{k}$ involving the valence orbital electrons or by $\operatorname{Racah}$ parameters $A, B$ and $C$, some specific relations between them being

$$
B=F_{2}-5 F_{4},
$$

and $\quad C=35 F_{4}$.

Though the experimental Slater-Condon integrals $F_{k}$ and $G_{k}$ for different oxidation states of the various transition ions have been obtained by many authors 
(Hinze and Jaffe 1963 ; Di Sipio et al 1970 ; Tondello et al 1970) the latter studies of Tondello et al are the best since they fulfilled certain theoretical conditions such as the inclusion of configuration interaction, linear variation of $F$ and $G$ as a function of atomic number, etc. However, the nephelauxetic or cloud-expanding effect (Jorgensen 1962 ; Dunn et al 1965 ; Lever 1968) describes the phenomenological lowering of these electron repulsion integrals of the transition ions in complexes as compared to their values in the free gaseous ions.

The present work deals with the comparison of the experimentally obtained spectroscopic Racah parameter $B$ of the complexes with the theoretically calculated ones using our MO szheme. We have considered here some weak-crystalline field tetrahedral and octahedral complexes, the energy states of which are nothing but crystal-field perturbed free ion states (Ballhausen 1962; Schlafer and Gliemann 1969). The nephelauxetic effect occurs due to two simultaneously occurring processes; one is due to a reduction in the effective charge on the metal on the approach of ligand electrons with a consequent expansion of the metal wave function and reduction in the degree of electron repulsions between the electrons of these orbitals and the other is due to the formation of molecular -orbitals delocalised on to the ligands representing the bonding covalency effects. To be more specific the Racah parameter $B$ compares such interactions as

$$
K\left(d_{e z}, d_{\sigma^{2}-y^{2}}\right)=\left(x z, x^{2}-y^{2} / x z, x^{2}-y^{2}\right),
$$

which for octahedral complexes takes the form

$$
K\left(t_{2 \sigma}(x z), e_{g}\left(x^{2}-y^{2}\right)\right)=\left[t_{2 \sigma}(x z), e_{g}\left(x^{2}-y^{2}\right) / t_{2 \sigma}(x z), e_{g}\left(x^{2}-y^{2}\right)\right],
$$

with a corresponding expression for tetrahedral molecules. In the above equations $d_{t z z}$ and $d_{\sigma^{2}-z^{2}}$ represent the respective orbitals in the free ion while $t_{2 \sigma}(x z)$ and $e_{g}\left(x^{2}-y^{2}\right)$ are the molezular orbitals in an octahedral crystal field namely,

$$
\begin{aligned}
& t_{2 \phi}(x z)=a d_{x z}^{*}+b \phi \text { ligands, } \\
& e_{0}\left(x^{2}-y^{2}\right)=c d_{x^{2}-y^{2}}^{*}+d^{\prime} \phi \text { ligands, }
\end{aligned}
$$

The above wave functions represent both the mechanisms of the nephelauxetic effect. The primes on $d_{x z}^{\prime}$ and $d_{z^{2}-y^{2}}^{\prime}$ indicate that these $d$-orbitals are not those of the free ion, namely, $d_{e s}$ and $d_{0^{2}-y^{2}}$ referred to in equation (2) but those corresponding to the self-consistent charge $z^{\prime}$ at the metal obtained in the complex reflecting the first central field covalency part of the nephelauxetic effect. The second bonding covalency part is taken care of in relation (3) by considering the $t_{2 o}$ and $e_{g}$ MO's of equation (4) instead of the pure $d$ orbitals to define these integrals for complexes.

The $B$ values of complexes extracted from their observable and identifiable $d-d$ bands will be compared with the calculated ones from the MO scheme. Also, greater the reduction in $B$ represented by the ratio

$$
B_{\text {complex }} / B_{\text {free 10n }}=\beta \text {, }
$$

the greater the covalency in the metal ligand bond and smaller the effective charge experienced by the $d$ electrons. Such trends in the $\beta$ values for a series of complexes containing various transition metals and ligands may also reveal the covalency variation. 


\section{The molecular orbital analysis of the complexes}

\subsection{The computational details}

For performing an all-valence electron calculation on these large systems involving first-row transition metal atoms we have used the unrestricted Roothaan-HartreeFock scheme which is set up at the INDO level of approximation following the parametrisation scheme of Clack et al (1972) and Clack (1974). Five $3 d$, one $4 s$ and three $4 p$ Slater-type orbitals from the metal atom contribute to the valence basis set with two separate $\zeta$ exponents to differentiate the $3 d$ with $n=3$ from the $4 s, 4 p$ orbitals with $n=4$. The $\zeta$ exponents of Zerner and Gouterman (1966) and Karlsson and Zerner (1973) have been adopted for the Slater orbitals of the metal tons. The valence $2 s, 2 p$ and $3 s, 3 p$ orbitals of the I and II row ligand atoms have also been represented by Slater-type orbitals, but with a single exponent $\zeta$ just characteristic of these atoms as was done by Pople, Santry and others (Pople and Beveridge 1976; Murrell and Harget 1972; Pople et al 1965; Pople and Segal 1966; Santry and Segal 1967). The coulombic $\gamma$ integrals $(\mu \mu / \nu v)$ among the valence electrons were theoretically worked out from the above valence Slater orbitals. The empirical parameter $\beta$ appearing in the off-diagonal core term calibrated by Clack et al (1972) as two separate $\beta_{9 d}$ and $\beta_{4 s,}$, for use with the Gouterman's exponents and his experiment based sum of ionisation potential and electron affinity have both been exploited for the transition metal atom. The additional correction necessary for the introduction of the one-centre exchanes integrals at the INDO level can be expressed in terms of the experimentally available SlaterCondon $F_{k}$ and $G_{k}$ integrals, which for the metal atom are assumed once again from the studies of Tondello et al (1967). The $B$ values of Tondello et al for the various oxidation states of the first transition atoms are given in table 1 . All the above parameters for the lighter ligand members have been taken from the studies of Pople, Santry and others (Pople and Beveridge 1976; Murrell and Harget 1972; Pople et al 1965; Pople and Segal 1966; Santry and Segal 1967). The $F_{k}, G_{k}$ exchange integrals employed for the II row chlorine atom are those of Benson and Hudson (1970).

Calculations have been performed on simple I row high spin transition metal halides (chlorides and fluorides) of both the tetrahedral and octahedral geometry.

Table 1. Racah integral $B$ (in $\mathrm{cm}^{-1}$ ) for atoms and ions of the first transition period, derived from the corresponding Oleari's Slater-Condon integrals.

\begin{tabular}{|c|c|c|c|c|c|c|c|}
\hline & $\mathbf{T i}$ & $\mathbf{v}$ & $\mathrm{Cr}$ & $\mathbf{M n}$ & $\mathbf{F e}$ & Co & $\mathrm{Ni}$ \\
\hline $\begin{array}{l}B(-1) \\
B(0) \\
B(+1) \\
B(+2) \\
B(+3)\end{array}$ & $\begin{array}{c}417 \cdot 5 \\
490 \\
560 \\
675 \\
\ldots\end{array}$ & $\begin{array}{c}502 \cdot 5 \\
572 \cdot 5 \\
642 \cdot 5 \\
751 \cdot 5 \\
(875 \cdot 1)^{*}\end{array}$ & $\begin{array}{c}585 \\
655 \\
725 \\
828 \\
(951 \cdot 1)\end{array}$ & $\begin{array}{l}670 \\
737 \cdot 5 \\
807 \cdot 5 \\
905 \\
\ldots\end{array}$ & $\begin{array}{c}750 \\
820 \\
890 \\
981 \cdot 5 \\
(1097 \cdot 5)\end{array}$ & $\begin{array}{c}835 \\
902 \cdot 5 \\
972 \cdot 5 \\
1058 \cdot 5 \\
(1177 \cdot 5)\end{array}$ & $\begin{array}{c}897 \cdot 5 \\
985 \\
1055 \\
1135 \\
\ldots\end{array}$ \\
\hline
\end{tabular}


The species studied and their actual geometry are given in the upper halves of tables 2 and 3. For tetrahedral molecules wo have used the experimentally determined $x$-ray bond distances of the anions of some of the corresponding salts since the optical data also are from these lattices. However, in the case of octahedral systems, since we could not get sufficient structure parameters for the anions of the corresponding salts, though they have been used for studying the optical spectra, the bond distances have been assumed to be similar to those of the solid lattices $\mathrm{MF}_{3}$ and $\mathrm{MCl}_{3}$ (Wyckoff 1963). These experimental metal-halogen bond lengths assumed in our calculation and as presented in tables 2 and 3 are taken from other references (Colton and Canterford 1969; Wyckoff 1963; Fenske et al 1965; Fenske and Radtke 1968; Basch et al 1966; Basch and Gray 1976; Larson et al 1974).

A spin unrestricted INDO MO calculation was carried out on the above complex ions. Oleari's $F_{k}, G_{k}$ integrals for zero oxidation state had to be used in most cases since the final output charge at the metal was approximately zero. For systems with degenerate ground state as $\mathrm{NiCl}_{4}^{2-}, \mathrm{VCl}_{\mathrm{a}}^{3-}$ and $\mathrm{VF}_{\mathrm{a}}^{s-}$ a symmetryrestricted calculation was performed.

We also performed an initial optimisation of the geometry by studying the variation of the total energy as a function of the metal-halogen bond distance. The theoretical equilibrium bond length corresponding to the lowest total energy obtained in this calculation is listed below the experimental ones in these tables. While this type of optimisation scheme is understandable for independent molecular units like $\mathrm{TiCl}_{4}, \mathrm{VCl}_{4}$, etc., and can be compared with the experimental results in a justifiable manner, the same may not hold good for solid complexes since our calculations have not taken into account the field created by the counter ions in the crystal lattice. So it is preferable to resort to the results of calculations with the experimental bond distances for predicting their properties. Our sole idea of finding the theoretical lowest energy bond length is to justify the use of these experimental bond lengths for our calculations and this objective has been more than fulfilled by predicting reasonably good theoretical bond lengths which compare well with the experimental ones.

\subsection{Electronic configuration and energy level ordering}

The ground state electronic configuration predicted from our calculations and listed in tables 2 and 3 for these various complexes are identical to the earlier predictions by crystal field study (Ballhausen 1962) and other MO schemes (Fenske et al 1965; Fenske and Radtke 1968; Basch et al 1976; Basch and Gray 1976). But the ordering of the eigen MO's does not coincide with earlier studies and a good deal of shuffling has taken place. Besides due to the open-shell nature of the scheme, the filled-up major metal $3 d a$ MO's lie deep down among the set of filled $a$ orbitals instead of being the top-most filled orbitals where they are conventionally expected. Hence an attempt to correlate the ordering of energy levels with the crystal field model and also with other earlier MO scheme results will be a failure. It must however be pointed out that we do not have any problem in predicting the experimental observations, although the above mentioned fictitious eigen MO ordering pattern could not be reproduced. Thus for example, with the tetrahedral $\mathrm{FeCl}_{-}^{1}-\left(d^{\dagger}\right)$, we have obtained an ordering $t_{2}^{a}<e^{\alpha}$ for the filled major $d$ a MQ's 


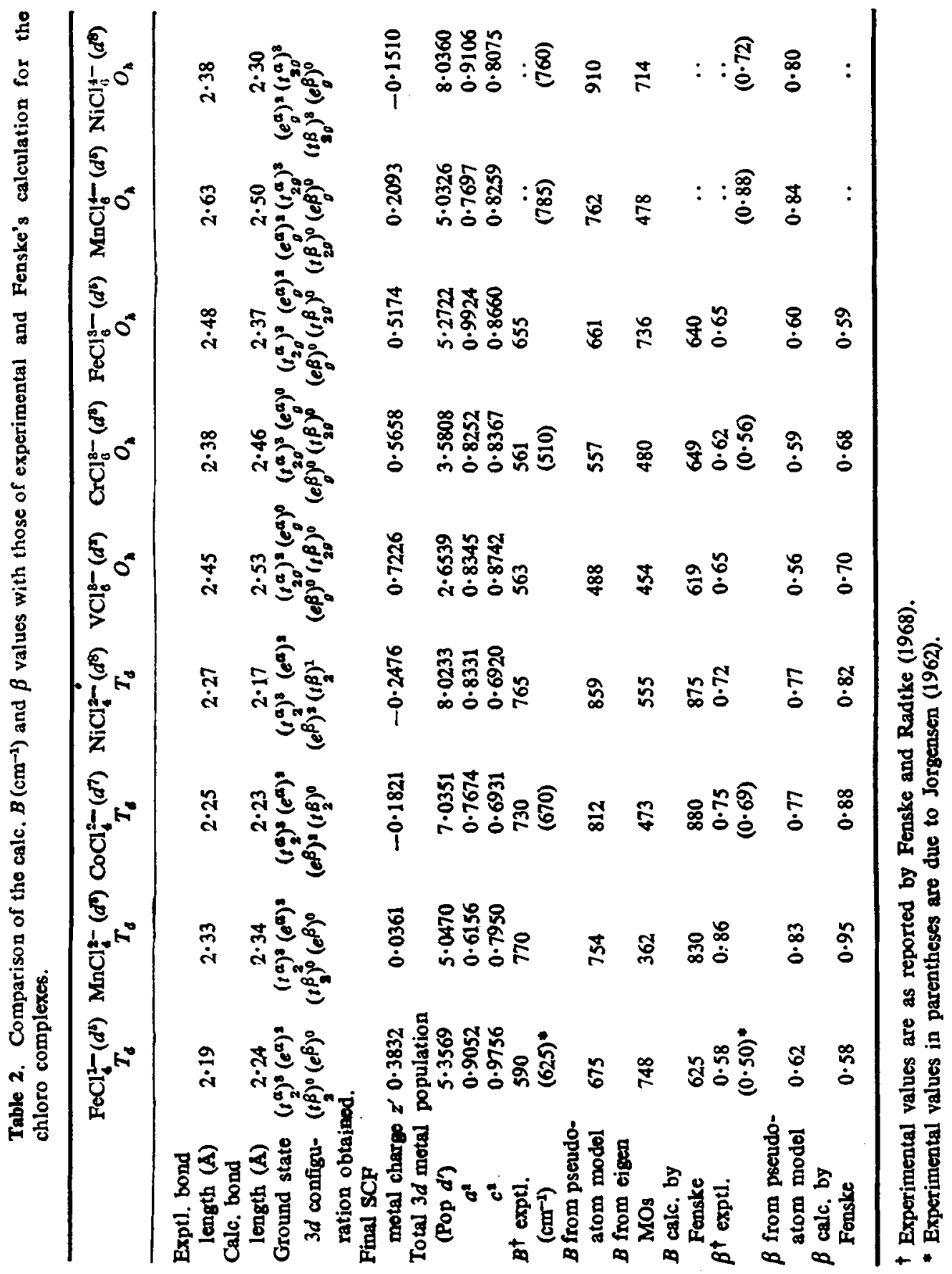


Table 3. Comparison of the calc. $B\left(\mathrm{~cm}^{-1}\right)$ and $\beta$ values with those of experio mental and Fenske's calculation for the fluoro complexes.

\begin{tabular}{|c|c|c|c|c|c|}
\hline & $\begin{array}{c}\mathrm{VF}_{6}^{3-\left(d^{2}\right)} \\
o_{h}\end{array}$ & $\begin{array}{c}\mathrm{CrF}_{\mathrm{e}}^{3-}\left(d^{\prime}\right) \\
O_{\mathrm{h}}\end{array}$ & $\begin{array}{c}\mathrm{FeF}_{6}^{3-}\left(d^{d}\right) \\
O_{\lambda}\end{array}$ & $\begin{array}{c}\mathrm{MnF}_{6}^{4-\left(d^{b}\right)} \\
O_{k}\end{array}$ & $\begin{array}{c}\mathrm{NiF}_{6}^{4-}\left(d^{8}\right) \\
O_{n}\end{array}$ \\
\hline Exptl. bond length $(\AA)$ & 1.94 & 1.93 & 1.91 & $2 \cdot 093$ & $2 \cdot 010$ \\
\hline CaIc. bond length $(\AA)$ & $2 \cdot 21$ & $2 \cdot 15$ & $2 \cdot 07$ & $2 \cdot 22$ & 1.985 \\
\hline $\begin{array}{l}\text { Ground state } 3 d \text { configura- } \\
\text { tion obtained. }\end{array}$ & $\begin{array}{l}\left(t_{20}^{a}\right)^{2}\left(e_{g}^{a}\right)^{0} \\
\left(t_{20}^{\beta}\right)^{0}\left(e_{g}^{\beta}\right)^{0}\end{array}$ & $\begin{array}{l}\left(t_{2 g}^{a}\right)^{8}\left(e^{a}\right)^{0} \\
\left.(t \beta)^{\beta}\right)^{0}\left(e^{\beta \beta}\right)^{0}\end{array}$ & $\begin{array}{l}\left(e_{0}^{\alpha}\right)^{2}\left(t_{20}^{a}\right)^{3} \\
\left(t_{20}^{\beta}\right)^{0}\left(e_{0}^{\beta B}\right)^{0}\end{array}$ & $\begin{array}{l}\left(t_{2 g}^{a}\right)^{3}\left(e_{g}^{a}\right)^{2} \\
\left(t_{2 g}^{B}\right)^{0}\left(e \beta_{g}\right)^{0}\end{array}$ & $\begin{array}{l}\left(t_{20}^{a}\right)^{3}\left(e_{g}^{a}\right)^{2} \\
\left(\beta_{2 g}^{\beta}\right)^{8}(e \beta)^{0}\end{array}$ \\
\hline $\begin{array}{l}\text { Final SCF metal charge } z^{\prime} \\
\text { Total } 3 d \text { metal population }\end{array}$ & 0.6890 & 0.6293 & 0.6481 & 0.2151 & -0.0246 \\
\hline$\left(\right.$ Pop $\left.d^{\prime}\right)$ & $2 \cdot 8286$ & $3 \cdot 7278$ & $5 \cdot 4266$ & $5 \cdot 1007$ & 8.0676 \\
\hline$a^{2}$ & 0.9030 & $0 \cdot 7340$ & 0.9018 & 0.9448 & 0.9435 \\
\hline$c^{2}$ & $0 \cdot 8317$ & $0 \cdot 8203$ & $0 \cdot 7216$ & 0.8049 & 0.7800 \\
\hline$B^{\dagger}$ exptl. $\left(\mathrm{cm}^{-1}\right)$ & 648 & $\begin{array}{c}680 \\
(820) *\end{array}$ & $\begin{array}{r}844 \\
(845)\end{array}$ & $(845)$ & $(960)$ \\
\hline$B$ from pseudo-atom model & 552 & 607 & 708 & 783 & 987 \\
\hline$B$ from eigen $\mathrm{MOs}$ & 466 & 421 & 563 & 572 & 723 \\
\hline$B$ calc. by Fenske & 689 & 712 & 694 & .. & .. \\
\hline$\beta^{\dagger}$ exptl. & $0 \cdot 75$ & $\begin{array}{l}0.74 \\
(0.89) *\end{array}$ & $\begin{array}{r}0.77 \\
(0.78)\end{array}$ & $(0 \cdot \ddot{94})$ & $(0 \cdot \ddot{91})$ \\
\hline$\beta$ from psendo-atom model & 0.63 & 0.64 & $0 \cdot 64$ & 0.87 & $0 \cdot 87$ \\
\hline$\beta$ calc. by Fenske & 0.77 & 0.74 & 0.64 & .. & . \\
\hline
\end{tabular}

t Experimental values are as reported by Fenske et al (1965).

* Experimental values in parantheses are due to Jorgensen (1962).

and $t_{2}^{\beta}<e^{\beta}$ for the corresponding unfilled $\beta$ MO's contrary to the crystal field prediction $t_{2}>e$. However, the calculation on the tetrahedral complex $\mathrm{FeCl}_{-2}^{2-}\left(d^{6}\right)$ with one more electron added to the earlier system arrived at the correct configuration $\left(t_{2}^{\alpha}\right)^{3}\left(e^{\alpha}\right)^{2}\left(e^{\beta}\right)^{1}\left(t_{2}^{\beta}\right)^{0}$ with the sixth electron going to the $e^{\beta}$ orbital in complete conformity with the simple crystal field model.

\section{Theoretical schemes for the evaluation of $B$}

In the present work we have theoretically evaluated $B_{\text {comples }}$ from the above results of our INDO MO analysis by two different schemes to be compared with the corresponding experimental results.

\subsection{The pseudo-atom model (scheme 1)}

Here the value for $B$ has been evaluated for the chloro and fluoro complexes by making use of the fact that its spectroscopic bands correspond to an independent pseudo-atom with charge $z^{\prime}$ which is finally obtained at the metal from the MO analysis. The $B\left(z^{\prime}\right)$ value corresponding to this charge $z^{\prime}$ is obtained by interpolation of the various $B$ values experimentally available for the different oxidation states of the free metal ions from Oleari et al found in table 3. Besides, 
because of the covalent mixing of the $d$ orbitals with the ligand orbitals in complexes, the orbitals lose their pure atomic nature and the valence electrons instead of being localised at atoms are slightly smeared out over the other atoms of the system as well. Hence, when working out the $B_{\text {comples, }}$ the new $3 d$ gross population termed as Pop $\left(d^{\prime}\right)$ at the metal in the complex which can be obtained from our MO analysis has to be considered instead of the corresponding Pop (d) of the free ion having charge $z^{\prime}$. First of all, the interpolated $B\left(z^{\prime}\right)$ value takes care of the first effect, namely, the contraction or expansion of metal orbitals due to changes in the effective charges on the metal as a result of electron donation via chemical bonding (the central field covalency part). Since $B$ involves electrostatic integrals among these $3 d$ electrons the second covalency effect is taken care of through a multiplication scaling factor [Pop $\left.\left(d^{\prime}\right) / \operatorname{Pop}(d)\right]^{2}$ involving the $3 d$ gross populations in the complex and free ion. Hence the pseudo-atom model suggests the formula

$$
B_{\text {eomplex }}=B\left(z^{\prime}\right)\left[\operatorname{Pop}\left(d^{\prime}\right) / \operatorname{Pop}(d)\right]^{2}
$$

\subsection{From the eigen MO's (scheme 2)}

Again the first effect due to the reduction in charge at the metal is taken care of through the interpolated $B\left(z^{\prime}\right)$ value corresponding to the final self-consistent charge $z^{\prime}$. Now to ascommodate the second effect of covalent mixing of the $d$ orbitals with the ligand orbitals, i.e., to account for delocalisation of the metal $d$ electrons on to ligands as mentioned earlier in the pseudo-atom scheme we refer back to the molecular orbitals in equations (4a) and (4b). Since $B$ involves integrals among these $3 d$ molecular orbitals the free ion $B\left(z^{\prime}\right)$ value corresponding to the purely metallic $d$ orbitals with $a=c=1$ has to be corrected by multiplying with the corresponding coefficients of the metal orbitals in the molecular orbitals namely, by the factor $a^{2} c^{2}$. Thus we derive the equation

$$
B_{\text {complex }}=B\left(z^{\prime}\right) a^{2} c^{2}
$$

for eigen MO's $t_{2 \sigma}$ and $e_{g}$ of octahedral (or $t_{2}$ and $e$ of tetrahedral) cases in accordance with Fenske's formulation (Fenske et al 1965).

\section{Discussion of results and conclusion}

The concept of differential covalency (Clack and Monshi 1977) may be worth mentioning here. Though the first effect -the effect of effective nuclear charge on the radial function is going to be the same for all the five $d$ orbitals, considering the covalent bonding of these $d$ orbitals with the ligand orbitals in the complex, all of them are not equally affected as can be seen from the differences in the metal coefficients $a$ and $c$ in the MOs which are not equal even for these highly symmetric molecules. Thus while for the free ion with pure $d$ orbitals with $a=c=1$ we have a unique $B$, the $B$ of the complex depends upon the orbitals involved in defining the $B$ integral. Thus we have several values for $B_{\text {eomplex }}$ corresponding to different $d$ orbital combinations and this must be correctly accounted for when deriving out $B$ from experimental and theoretical sides. This is amply exempli- 
fied by the disagreement in the reported experimental $B$ values for the same complex by different authors as can be seen from tables 2 and 3 . It may be due to the ' differential covalency ' as each one of them might have resorted to different bands to get the value of the $B$ for the same complex. By equation (7) we just arrive at one typical $B$ value for the complex involving $t_{2 p}$ and $e_{0}$ (or $t_{2}$ and $e$ ) orbitals. In a spin-unrestricted scheme like ours, the coefficients of the $a$ and $\beta$ sets of the MO's may themselves differ. So in trying to compare the calculated results with the experimental ones, one must be doubly careful in choosing the appropriate value for $a^{2}$ and $c^{2}$ when trying to calculate $B$ theoretically.

The multiplication factor $\left(z^{\prime}+2\right) /\left(z^{\prime}+3\right)$ included by Fenske et al $(1965)$ in their formula was invoked to bring down the calculated values nearer to experimental ones, as their calculated $B_{\text {complex }}$ came out to be too high due to using theoretical $B$ (sec.), i.e., theoretical $B\left(z^{\prime}\right)$. Even when we tried using the theoretical $B(0)$ available for zero oxidation state Watson's function (Watson 1960) (which corresponds overlapwise to the Gouterman orbitals used here), the $B_{\text {complex }}$ came out as large and might have needed such a correction factor as $\left(z^{\prime}+2\right) /\left(z^{\prime}+3\right)$. However, since we resorted to the best recent Di Sipio's experimental $B$ values of the free ions for getting the interpolated $B(z)$, no such arbitrary correction term is necessary.

The $B_{\text {complex }}$ thus evaluated from both the schemes for the chlorides and fluorides are compared with the experimental values in tables 2 and 3 . On the whole the pseudo-atom model fares far better. It must be noted that the pseudoatom model gives us an average $B$ value for the complex as this does not differentiate among the orbitals and $B$ is just a function of the final charge $z^{\prime}$ and gross $3 d$ population.

But the values of $B$ calculated from the eigen MOs are extremely poor compared to the predictions of the pseudo-atom scheme. There may be two possible reasons for this :

(i) the goodness of each individual eigenfunction $t_{20}, e_{0}$ or $t_{2}, e$ obtained by using the INDO approximation is not sufficient for this specific purpose of calculating a multiorbital hybrid integral of the form $\left(z^{2}, x z / x y, y z\right)$;

(ii) the second scheme from eigen MOs badly fails in cases where the calculated self-consistent charge $z^{\prime}$ at the metal is far from being zero.

It may be remembered that we have adopted Gouterman's neutral atom exponents for the metal. Even though the ideal path is to optimise the exponents as well along with the LCAO MO coefficients in the self-consistent cycle, one should at least resort to suitable basis functions comparable to the resultant charge $z^{\prime}$ of the metal. Otherwise, the eigenvectors obtained, i.e., the coefficients $a$ and $c$ may not be good enough to be used along with the interpolated $B\left(z^{\prime}\right)$ value, since they correspond to basis functions of zero oxidation state. The above two factors may account for the failure of the scheme from the eigen MO's. The pseudo-atom which depends upon the final charge and gross $3 d$ population can surpass the above drawbacks as it rests on the validity of the total gross effocts which need not be as badly affected as the individual eigenvectors by the various approximations like the INDO and unsuitable basis set. Even the results from the pseudo-atom model can also be further improved towards excellent agreement with experiment by the adoption 
of suitable metal basis functions to suit the final charge since the same problem provails here too, though to a lesser extent. In general it can be noticed that the pseudo-atom values are higher or lower from experimental results depending upon whether the final charge $z^{\prime}$ is negative or positive. Hence the incorporation of a slightly expanded or contracted basis set respectively for the first and second cases in conformity with their charge $z^{\prime}$ might bring the calculated $B_{\text {complex }}$ values closer to the experimental results. But as we do not have single Slater exponents for higher oxidation states and negative ions of metal although such multi Richardson's functions (Richardson et al 1962,1963) are available, an attempt in this direction could not be made by us with our MO programme. Hence there is a nocessity to develop such single $\zeta$ metal exponents for other oxidation states along with their corresponding $\beta$ parameters. On the whole the results of the pseudo-atom model are reasonably good as can be seen and in many cases better than those of Fenske's which are also included in these tables for comparison.

Nephelauxetic series predict that the $\mathrm{Cl}^{-}$ion is more covalent and hence capable

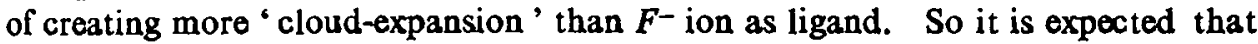
the calculated $B_{\text {comblex }}$ value as well as the experimental one for the same metal ion with a particular formal charge must be less for chloro complexes than for the fluoro complexes. This has been beautifully borne out by calculation and experi-

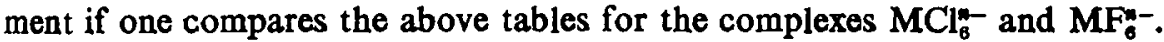

The covalency factor $\beta$ has also been worked out using equation (5) from tho $B_{\text {compler }}$ calculated through the better pseudo-atom scheme alone, and the $B_{\text {free }}$ 10: experimental value as compiled in table 1 . This $\beta$ is compared with the experimental values and with those reported by Fenske et al (1965) and Fenske and Radtke (1968) in the lower halves of tables 2 and 3 . It can be seen that this $\beta$ also reflects the concept of differential covalency as the experimental values reported by different authors for the same species differ considerably, a fact which again need not be due to the experimental discrepancies. The $\beta$ worked out by us from our calculated $B_{\text {complax }}$ of pseudo-atom approach is again an average value for a complex and does not take care of differential covalency.

In general the agreement between the experimental and calculated ones has been far better than Fenske's results in the case of chloro complexes. Fenske's values aro generally better in the case of fluoro complexes. Failure in our cases must be due to poor basis set for the metal orbitals as we have obtained heavy positive charge nearing unity at the metal in majority of these cases as in the octahedral $\mathrm{MF}_{6}^{\text {s- }}$ systems.

Finally since any spectroscopic crystal-field transition corresponds to just the total energy difference of the system before and after a specific electronic transition among two $d$ orbitals, one can at the most get only an average value for each of the individual Racah or Slater-Condon integrals of complexes from available spectroscopic transitions. We have theoretically suggested several possible values for $B$ in complex depending upon the $d$ orbital combinations involved from the concept of differential covalency. However, it is impossible to extract all those out from the observable and identifiable $d-d$ bands that are available since the number of unknowns to be solved will be larger than the number of equations that can be provided by experimental transition energies. The outcome from our pseudo-atom model is a reasonably good theoretical counterpart to becompared with the reported 
experimental average values and the second formula (equation (7)) from the eigen MOs (scheme 2) is less convincing in this context.

\section{Acknowledgements}

The au thors wish to thank the Council of Scientific and Industrial Research, New Delhi, for a fellowship to SV.

\section{References}

Ballhausen C J 1962 Introduction to ligand field theory (New York : McGraw-Hill)

Basch H and Gray H B 1967 Inorg. Chem. 6365

Basch H, Viste A and Gray H B $1966 \mathrm{~J}$. Chem. Phys. 4410

Benson H G and Hudson A 1970 Theor. Chim. Acta 23259

Clack D W 1974 Mol. Phys. 271513

Clack D W, Hush N S and Yandle J R 1972 J. Chem. Phys. 573503

Clack D W and Monshi M 1977 Inorg. Chim. Acta 22261

Colton R and Canterford J H 1969 Halides of the first row transition metals (New York : Wiley-Interscience)

Condon E U and Shortley G H 1959 The theory of atomic spectra (Cambridge: University Press)

Di Sipio L, Tondello E, De Michelis G and Oleari L 1970 Inorg. Chem. 9927

Dunn T M, McClure D S and Pearson R G 1965 Some aspects of crystal field theory (New York : Harper and Row)

Fenske R F, Caulton K G, Radtke D D and Sweeney C C 1965 Inorg. Chem. 5960

Fenske R F and Radtke D D 1968 Inorg. Chem. 7479

Griffith J S 1971 The theory of transition metal ions (Cambridge: University Press)

Hinze J and Jaffe H H $1963 \mathrm{~J}$. Chem. Phys. 381834

Jorgensen C K 1962 Absorption spectra and chemical bonding in complexes (Oxford, London : Pergamon Press)

Karkson G and Zerner M 1973 Int. J. Quantum Chem. S8 145

Larson S, Viinikka E K and Cannolly J 1974 Int. J. Quantum Chem. S8 145

Lever A B P 1968 Inorganic electronic spectroscopy (Amsterdam: Elsevier)

Murrell J N and Harget A J 1972 Semi-empirical self-consistent field molecular orbital theory of molecules (London: Wiley-Interscience)

Pople J A and Beveridge D L 1976 Approximate molecular orbital theory (New York : McGrawHill)

Pople J A, Santry D P and Segal G A 1965 J. Chem. Phys. 43 S136

Pople J A and Segal G A 1966 J. Chem. Phys. 443289

Richardson J W, Nieuport W C, Powell R R and Edgell W F 1962 J. Chem. Phys. 361057

Richardson J W, Nieuport W C, Powell R R and Edgell W F 1963 J. Chem. Phys. 38796

Santry D' and Segal G A 1967 J. Chem. Phys. 47158

Schlafer H L and Gliemann G 1969 Basic principles of ligand field theory (New York: WileyInterscience)

Slater J C 1960 Quantum theory of atomic structure (New York : McGraw-Hill)

Tondello E, De Michelis G, Oleari L and Di Sipio L 1967 Coord. Chem. Rev. 265

Watson R E 1960 Phys. Rev. 1191934

Wyckoff R W G 1963 Crystal structures (New York: Interscience)

Zerner M and Gouterman M 1966 Theor. Chim. Acta 444 